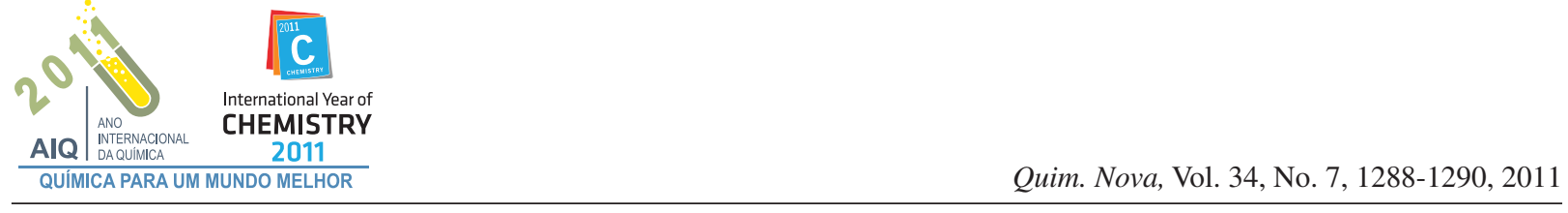

\title{
UM MÉTODO PARA AVALIAR A TAXA DE CORROSÃO
}

\section{Ednilson Luiz Silva Vaz, Heloisa Andréa Acciari e Eduardo Norberto Codaro*}

Departamento de Física e Química, Faculdade de Engenharia de Guaratinguetá, Universidade Estadual Paulista, Av. Dr. Ariberto Pereira da Cunha, 333, 12516-410 Guaratinguetá - SP, Brasil

Recebido em 2/9/10; aceito em 10/2/11; publicado na web em 15/4/11

\begin{abstract}
A METHOD FOR CORROSION RATE EVALUATION. In this paper it is proposed an indirect method to evaluate the corrosion rate of an aluminum and zinc alloy in alkaline solution by using a well-known device for collecting gases over water. The hydrogen gas formation, a corrosion product, is monitored at different time intervals and associated with the alloy mass loss. It has been suggested that the students should work in groups, which may make feasible the social interaction among them and that results discussion may be done collectively under a professor orientation. This proposal may propitiate the learning of terminology and involved concepts as well as contribute to a better understanding of corrosion phenomena that occur in their everyday life.
\end{abstract}

Keywords: chemistry education; corrosion; social interaction.

\section{INTRODUÇÃO}

A corrosão pode ser explicada como a deterioração do material metálico que se inicia na sua superfície por ação química ou eletroquímica de um meio oxidante, agravada geralmente por esforços mecânicos. Muitos métodos são utilizados para avaliar a corrosão, no entanto, somente alguns deles estão normalizados. Destes, os mais conhecidos e provavelmente os mais aceitos pela indústria são os ensaios de exposição à névoa salina e por imersão. ${ }^{1,2}$ A resistência à corrosão e a velocidade ou taxa de corrosão de um material metálico são os parâmetros mais importantes a serem determinados. Enquanto a primeira fornece uma informação meramente comparativa da estabilidade de dois ou mais materiais num mesmo meio ou de um material em dois ou mais meios, a segunda apresenta informação da rapidez com que a reação de corrosão ocorre. Cabe mencionar que esses parâmetros são determinados geralmente mediante ensaios de curta duração que tentam simular as condições reais às quais serão expostos os materiais em serviço. Portanto, é necessária certa precaução quando se pretende selecionar um material estimando sua vida útil por extrapolação desses parâmetros. ${ }^{3,4}$

Neste contexto, propõe-se uma experiência de química, na qual ocorre um processo de corrosão entre um material metálico e um meio agressivo, com o objetivo de transmitir conhecimento e propiciar ao aluno a articulação entre os conceitos envolvidos e os fenômenos de corrosão que espontaneamente ocorrem no seu dia-a-dia. Em particular, será avaliada a taxa de corrosão de uma liga de alumínio e zinco (Al 89\% e $\mathrm{Zn} \mathrm{6 \% )} \mathrm{em} \mathrm{solução} \mathrm{de} \mathrm{NaOH}$ 2,0 mol/L, acompanhando a formação e coleta de gás $\mathrm{H}_{2}$ sobre água. Esse conteúdo está vinculado ao tema estruturador "Transformações químicas no dia-a-dia" sugerido pela SEMTEC. ${ }^{5}$

\section{FUNDAMENTAÇÃO TEÓRICA}

Por ser a reação de corrosão uma reação redox, são também aplicáveis os métodos empregados nos estudos cinéticos baseados no acompanhamento do consumo de reagentes ou da formação de

*e-mail: codaro@ feg.unesp.br produtos em diferentes intervalos de tempo. ${ }^{6}$ Uma prática frequente num laboratório é a determinação da quantidade de gás liberado por uma reação química. Alguns gases podem ser coletados sobre água num dispositivo que utiliza uma proveta invertida, como o mostrado na Figura 1. Esses gases não devem reagir com a água e ser, praticamente, insolúveis nela, como nos casos do $\mathrm{H}_{2}$ e do $\mathrm{O}_{2}$ cujas solubilidades a $25^{\circ} \mathrm{C}$ são 1,91 e $3,16 \mathrm{~cm}^{3} / 100 \mathrm{~cm}^{3}$, respectivamente. ${ }^{7} \mathrm{O}$ volume de gás coletado é medido levantando ou abaixando a proveta quanto necessário até que os níveis de água dentro e fora dela sejam iguais. Desta maneira, é suprimida a pressão hidrostática e a pressão total dos gases, hidrogênio mais vapor de água, dentro da proveta será igual à pressão atmosférica, de acordo com a Lei de Dalton das pressões parciais. Como a pressão de vapor da água depende apenas da temperatura e esta permanece constante durante o experimento, a pressão do gás também permanecerá constante. Segundo a Lei do Gás Ideal, o volume lido na proveta é diretamente proporcional ao número de mols de gás produzidos pela reação química, em nosso caso, $\mathrm{o} \mathrm{H}_{2}$ produzido pela corrosão da liga de $\mathrm{Al} \mathrm{em} \mathrm{NaOH} \mathrm{2,0} \mathrm{mol/L.}$ Portanto, quanto mais rápido o desprendimento de gás maior será a taxa de corrosão.

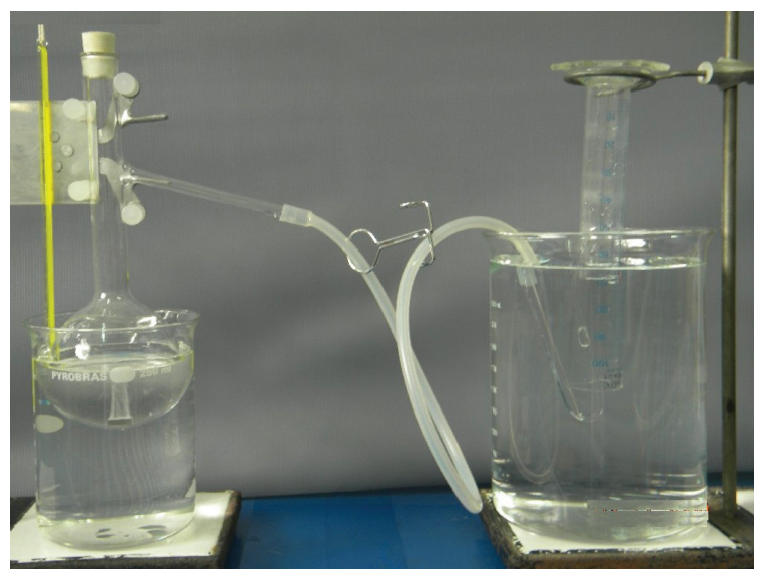

Figura 1. Dispositivo para coleta de gás $\mathrm{H}_{2}$ 


\section{PARTE EXPERIMENTAL}

Os materiais e reagentes necessários para que cada grupo realize o referido experimento são: 1 corpo-de-prova em forma de paralelepípedo de aproximadamente $1,5 \times 0,5 \times 0,5 \mathrm{~cm} ; 1$ lixa d'água $\mathrm{n}^{\circ}$ 80; 2 pissetas, uma com água destilada e outra com álcool; 1 pinça metálica; 1 secador de cabelos; 1 paquímetro ou régua; 1 balança analítica; 2 suportes; 2 garras; 1 balão de Engler de $250 \mathrm{~mL}$ com rolha; 1 mangueira de látex; 1 tubo de vidro em U; 1 pinça de Mohr; 2 béqueres, um de 1000 e outro de $3000 \mathrm{~mL} ; 2$ provetas, uma de 100 e outra de $250 \mathrm{~mL} ; 1$ vidro de relógio; 1 termômetro de 0 a $100{ }^{\circ} \mathrm{C} ; 1$ cronômetro; 1 funil de vidro e solução de $\mathrm{NaOH}$ 2,0 mol/L.

\section{Metodologia}

Para a realização do experimento é necessário formar de 4 a 6 grupos de trabalho, de modo que, no final deste sejam obtidos gráficos que permitam a discussão coletiva da taxa de corrosão. Cada grupo trabalhará com 1 corpo-de-prova (c-d-p) e determinará a taxa de corrosão da liga no meio básico. A preparação da superfície do c-d-p será feita com o auxílio de uma lixa com o intuito de obter as seis faces do paralelepípedo fisicamente semelhantes. Após esse procedimento, manipular o c-d-p com uma pinça metálica e lavá-lo com água destilada e depois com álcool, secá-lo utilizando o secador de cabelo, pesá-lo em uma balança analítica e começar o preenchimento da Tabela 1. Com um paquímetro (ou régua), calcular a superfície exposta do c-d-p (S). Utilizando uma proveta, medir um volume de solução de $\mathrm{NaOH}$ 2,0 mol/L igual a 50 vezes a área exposta do c-dp. O fator 50 é para evitar a saturação da solução com produtos de corrosão durante o teste. Montar o aparelho conforme o da Figura 1. O procedimento segue com a adição da solução de $\mathrm{NaOH}$ dentro do balão com o auxílio de um funil. É necessário assegurar que o nível da água no béquer pequeno (banho) cubra $2 / 3$ do balão e que a proveta invertida não tenha ar. Introduzir o c-d-p no balão, fechá-lo rapidamente com a rolha e disparar o cronômetro. Observar que o c-d-p não reage imediatamente com o meio, senão logo após alguns minutos. $\mathrm{O}$ gás $\mathrm{H}_{2}$, produto da reação de corrosão, é coletado na proveta invertida deslocando a água ali contida. A cada $12 \mathrm{~min}$ fechar a mangueira com a pinça de Mohr e medir rapidamente o volume de gás coletado levantando ou abaixando a proveta até que os níveis de água dentro e fora dela sejam iguais. Finalizado o experimento após 72 min, retirar a rolha, transferir a solução para um frasco de resíduos e o c-d-p para um vidro de relógio, pegá-lo com a pinça, lavá-lo, secá-lo e pesá-lo como descrito anteriormente. Completar a Tabela 1 calculando a perda de massa total e a taxa média de corrosão.

\section{DISCUSSÃO}

A taxa média de corrosão em um processo de corrosão generalizada é frequentemente definida pela perda de massa do material metálico por unidade de superfície, num certo intervalo de tempo. ${ }^{4}$ $\mathrm{O}$ aluno intui que quanto maior for o volume de $\mathrm{H}_{2}$ coletado nos intervalos de tempo, maior será a taxa de corrosão da liga nesse meio. No entanto, o volume de gás coletado na unidade de tempo não é uma medida comumente usada para definir este parâmetro. Neste contexto, mediante uma transformação matemática que vincule o volume de $\mathrm{H}_{2}$ com a perda de massa da liga, é possível obter uma unidade mais apropriada da taxa de corrosão, no sentido de enfatizar o consumo do material no lugar do produto de sua corrosão. O c-dp está constituído basicamente por $\mathrm{Al}$ e Zn. É bem conhecido que esses metais são oxidados em meio fortemente alcalino produzindo $\mathrm{H}_{2}$, além de tetra-hidroxoaluminato(III) e tetra-hidroxozincato(II), respectivamente (Equações 1 e 2).
Tabela 1. Características do material, condições experimentais e parâmetros a serem obtidos

\begin{tabular}{lcc}
\hline Antes do ensaio & Valor & Unidade \\
\hline Massa inicial do paralelepípedo & $\mathrm{mg}$ \\
Superfície exposta do paralelepípedo $(\mathrm{S})$ & $\mathrm{cm}^{2}$ \\
Volume de NaOH 2,0 mol/L $(50 \mathrm{~S})$ & $\mathrm{cm}^{3}$ \\
Temperatura de trabalho & ${ }^{\circ} \mathrm{C}$ \\
Pressão atmosférica & $\mathrm{mmHg}$ \\
Durante o ensaio & Unidade \\
Volume de $\mathrm{H}_{2}$ coletado após $12 \min \left(\mathrm{V}_{1}\right)$ & $\mathrm{mL}$ \\
Volume de $\mathrm{H}_{2}$ coletado após $24 \min \left(\mathrm{V}_{2}\right)$ & $\mathrm{mL}$ \\
Volume de $\mathrm{H}_{2}$ coletado após $36 \min \left(\mathrm{V}_{3}\right)$ & $\mathrm{mL}$ \\
Volume de $\mathrm{H}_{2}$ coletado após $48 \min \left(\mathrm{V}_{4}\right)$ & $\mathrm{mL}$ \\
Volume de $\mathrm{H}_{2}$ coletado após $60 \min \left(\mathrm{V}_{5}\right)$ & $\mathrm{mL}$ \\
Volume de $\mathrm{H}_{2}$ coletado após $72 \min \left(\mathrm{V}_{6}\right)$ & $\mathrm{mL}$ \\
Após o ensaio & $\mathrm{Unidade}$ \\
Massa final do paralelepípedo & $\mathrm{mg}$ \\
Perda de massa: inicial - final $(\mathrm{P})$ & $\mathrm{mg}$ \\
Taxa média de corrosão (P/72) & $\mathrm{mg} / \mathrm{min}$ \\
\hline
\end{tabular}

$$
\begin{gathered}
2 \mathrm{Al}(\mathrm{s})+2 \mathrm{OH}^{-}(\mathrm{aq})+6 \mathrm{H}_{2} \mathrm{O}(\mathrm{l}) \rightarrow 2 \mathrm{Al}(\mathrm{OH})_{4}^{-}(\mathrm{aq})+3 \mathrm{H}_{2}(\mathrm{~g}) \\
\mathrm{Zn}(\mathrm{s})+2 \mathrm{OH}^{-}(\mathrm{aq})+2 \mathrm{H}_{2} \mathrm{O}(\mathrm{l}) \rightarrow \mathrm{Zn}(\mathrm{OH})_{4}{ }^{2-}(\mathrm{aq})+3 \mathrm{H}_{2}(\mathrm{~g})
\end{gathered}
$$

Portanto, a relação de matéria é:

$$
n \text { mols de } \mathrm{H}_{2}=\frac{X \text { g de } \mathrm{Al}\left(3 \mathrm{mols} \text { de } \mathrm{H}_{2}\right)}{54 \mathrm{~g} \mathrm{de} \mathrm{Al}}
$$

Pela equação que rege o comportamento dos gases ideais $\mathrm{P}$ V $=\mathrm{n} \mathrm{R} \mathrm{T}$, temos:

$$
\mathrm{V}_{\mathrm{H}_{2}}=\mathrm{X}\left(\frac{3}{54}\right)\left(\frac{\mathrm{RT}}{\mathrm{P}_{\mathrm{H}_{2}}}\right)=\mathrm{X}(\mathrm{K})
$$

onde K é uma constante, já que o experimento foi realizado a pressão e temperatura constantes. De modo geral, cada volume de $\mathrm{H}_{2}\left(\mathrm{~V}_{\mathrm{n}}\right)$ coletado na proveta é diretamente proporcional à massa dissolvida do material metálico ou, mais especificamente, à perda de massa do paralelepípedo $\left(\mathrm{P}_{\mathrm{n}}\right)$. Sendo assim:

$$
\frac{\mathrm{V}_{1}}{\mathrm{~V}_{n}}=\frac{\mathrm{P}_{1}}{\mathrm{P}_{n}} \operatorname{com~} \mathrm{n}=1,2, \ldots, 6
$$

Para $\mathrm{n}=6, \mathrm{~V}_{1}$ e $\mathrm{V}_{6}$ são o primeiro e o último volume de $\mathrm{H}_{2}$ coletado, $\mathrm{P}_{6}$ é a perda de massa total obtida no final do experimento $(\mathrm{P})$, portanto, $\mathrm{P}_{1}$ pode ser calculada. Assim, conhecendo $\mathrm{P}_{1}$ é possível calcular $\mathrm{P}_{2}, \mathrm{P}_{3}, \mathrm{P}_{4}$ e $\mathrm{P}_{5}$. Com um simples rearranjo matemático pode-se demonstrar que $\mathrm{P}_{\mathrm{n}}=\mathrm{P}_{6} \mathrm{~V}_{\mathrm{n}} / \mathrm{V}_{6}$, ou seja, $\mathrm{P}_{\mathrm{n}}$ será uma fração de P. A Tabela 2 resume este formalismo matemático e organiza a coleção de dados.

Cada grupo deve colocar num gráfico (perda de massa por unidade de superfície versus tempo) seus dados e traçar a melhor reta que passe entre os pontos. Na Figura 2a são mostrados dois conjuntos de valores experimentais, ajustados com duas retas cujos coeficientes angulares 
Tabela 2. Cálculo da perda de massa em função do volume de $\mathrm{H}_{2}$ coletado

\begin{tabular}{lccc}
\hline $\begin{array}{l}\text { Tempo } \\
(\mathrm{min})\end{array}$ & $\begin{array}{c}\text { Volume de } \mathrm{H}_{2} \\
(\mathrm{~mL})\end{array}$ & $\begin{array}{c}\text { Perda de massa } \\
(\mathrm{mg})\end{array}$ & $\begin{array}{c}\text { Perda de massa por unidade de } \\
\text { superfície }\left(\mathrm{mg} / \mathrm{cm}^{2}\right)\end{array}$ \\
\hline 12 & $\mathrm{~V}_{1}$ & $\mathrm{PV}_{1} / \mathrm{V}_{6}$ & $(\mathrm{P} / \mathrm{S})\left(\mathrm{V}_{1} / \mathrm{V}_{6}\right)$ \\
24 & $\mathrm{~V}_{2}$ & $\mathrm{PV}_{2} / \mathrm{V}_{6}$ & $(\mathrm{P} / \mathrm{S})\left(\mathrm{V}_{2} / \mathrm{V}_{6}\right)$ \\
36 & $\mathrm{~V}_{3}$ & $\mathrm{PV}_{3} / \mathrm{V}_{6}$ & $(\mathrm{P} / \mathrm{S})\left(\mathrm{V}_{3} / \mathrm{V}_{6}\right)$ \\
48 & $\mathrm{~V}_{4}$ & $\mathrm{PV}_{4} / \mathrm{V}_{6}$ & $(\mathrm{P} / \mathrm{S})\left(\mathrm{V}_{4} / \mathrm{V}_{6}\right)$ \\
60 & $\mathrm{~V}_{5}$ & $\mathrm{PV}_{5} / \mathrm{V}_{6}$ & $(\mathrm{P} / \mathrm{S})\left(\mathrm{V}_{5} / \mathrm{V}_{6}\right)$ \\
72 & $\mathrm{~V}_{6}$ & $\mathrm{PV}_{6} / \mathrm{V}_{6}$ & $(\mathrm{P} / \mathrm{S})\left(\mathrm{V}_{6} / \mathrm{V}_{6}\right)$ \\
\hline
\end{tabular}

(taxas médias de corrosão) e valores extrapolados a $\mathrm{P}=0$ são similares. Este gráfico permite obter as seguintes informações: a taxa de corrosão é constante em todo o intervalo de tempo do experimento; o tempo mínimo a $\mathrm{P}=0$ pode ser interpretado como o tempo necessário para a dissolução da camada de óxidos que protege a liga, como exemplificado nas Equações 6 e 7. De fato, foi possível observar que a reação parece não começar logo após a imersão do c-d-p; somente depois que a liga é atingida, as bolhas de gás $\mathrm{H}_{2}$ são visualizadas.

$$
\begin{gathered}
\mathrm{Al}_{2} \mathrm{O}_{3}(\mathrm{~s})+2 \mathrm{OH}^{-}(\mathrm{aq})+3 \mathrm{H}_{2} \mathrm{O}(\mathrm{l}) \rightarrow 2 \mathrm{Al}(\mathrm{OH})_{4}^{-}(\mathrm{aq}) \\
\mathrm{ZnO}(\mathrm{s})+2 \mathrm{OH}^{-}(\mathrm{aq})+\mathrm{H}_{2} \mathrm{O}(\mathrm{l}) \rightarrow \mathrm{Zn}(\mathrm{OH})_{4}{ }^{2-}(\mathrm{aq})
\end{gathered}
$$

A Figura 2b apresenta um gráfico com os resultados experimentais indicados na forma de perda de massa versus tempo, com intuito de evidenciar o efeito de diferentes superfícies expostas na velocidade de corrosão. Comparando este gráfico com o da Figura 2a, os alunos poderão visualizar claramente que diferentes taxas (coeficientes angulares) são uma consequência de se utilizar c-d-p com superfícies expostas diferentes. Isto está de acordo com um princípio fundamental da cinética química heterogênea "a velocidade de uma reação química heterogênea aumenta quando aumenta a área de contacto entre os reagentes".

\section{CONSIDERAÇÕES FINAIS}

Referente ao método, o experimento é simples, reprodutível e exequível no tempo de uma aula experimental ( 2 h), aplicável principalmente em $\mathrm{Al}, \mathrm{Zn}$ e ligas destes elementos que na presença de solução de $\mathrm{NaOH}$ liberam $\mathrm{H}_{2}$, extensivo a outros materiais e meios cuja reação libera $\mathrm{H}_{2}$, fornece um parâmetro importante dos processos corrosivos.

Referente à aprendizagem, propicia a interação social entre os alunos viabilizada pelo trabalho em grupo, permite a discussão dos resultados entre os diferentes grupos e abre espaço para que o
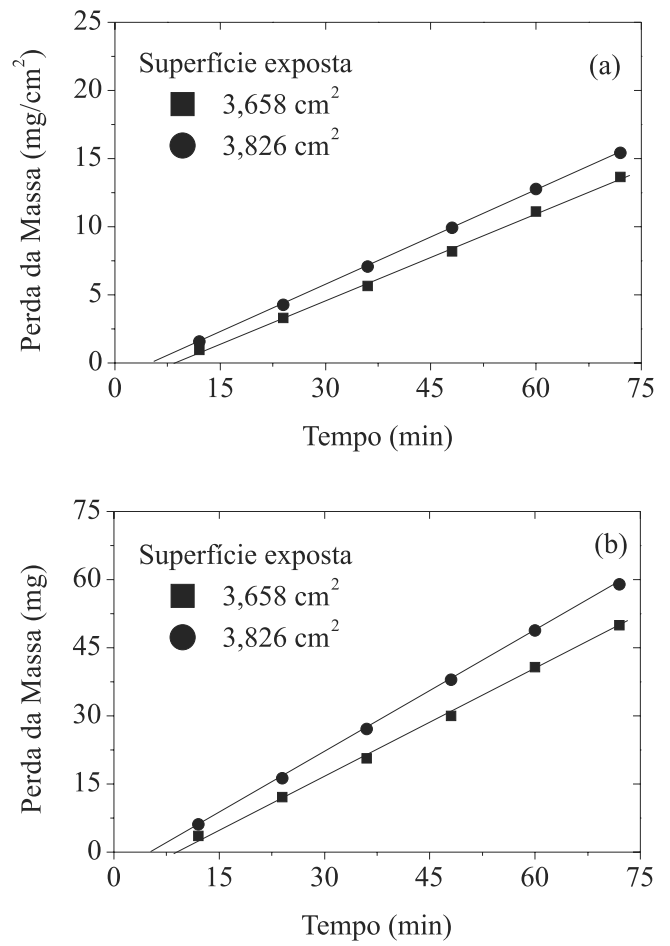

Figura 2. Determinação da taxa de corrosão (a) e efeito da superfície exposta do c-d-p na taxa de corrosão (b)

professor discuta com os alunos a respeito do fenômeno da corrosão como um processo natural que acontece no cotidiano.

\section{REFERENCIAS}

1. Standard Practice for Operating Salt Spray (fog) Apparatus; ASTM B117-07a, 2008, p. 1-10.

2. Standard Practice for Laboratory Immersion Corrosion Testing of Metals; ASTM G31-72, 1995, p. 95-101.

3. Gemelli, E.: Corrosão de Materiais Metálicos e sua Caracterização, $1^{\mathrm{a}}$ ed., LTC: Rio de Janeiro, 2001.

4. Gentil, V.: Corrosão, $5^{\text {a }}$ ed., LTC: Rio de Janeiro, 2007.

5. Secretaria de Educação Média e Tecnológica. Parâmetros Curriculares Nacionais + Ensino Médio: Orientações Educacionais Complementares aos Parâmetros Curriculares Nacionais - ciências da natureza, matemática e suas tecnologias, Brasília: MEC; SEMTEC, 2002, p. 144.

6. Brown, T. L.; Lemay, H. E.; Bursten, B. E.; Burdge, J. R.; Química: A Ciência Central, $9^{\mathrm{a}}$ ed., Pearson Education: São Paulo, 2005.

7. Handbook of Chemitry and Physics, 52 $2^{\text {nd }}$, CRC: Cleveland, 1972. 